\title{
Electronic structure, magnetism, and superconductivity in infinite-layer nickelates
}

\section{Electronic structure of the parent compound of superconducting infinite-layer nickelates}

Authors: M. Hepting, D. Li, C. J. Jia, H. Lu, E. Paris, Y. Tseng, X. Feng, M. Osada, E. Been, Y. Hikita, Y.-D. Chuang, Z. Hussain, K. J. Zhou, A. Nag, M.

Garcia-Fernandez, M. Rossi, H. Y. Huang, D. J. Huang, Z. X. Shen, T. Schmitt, H.

Y. Hwang, B. Moritz, J. Zaanen, T. P. Devereaux, and W. S. Lee

arXiv:1909.02678, to be published in Nature Materials

2. Model construction and a possibility of cuprate-like pairing in a new $d^{9}$ nickelate superconductor $(\mathrm{Nd}, \mathrm{Sr}) \mathrm{NiO}_{2}$

Authors: H. Sakakibara, H. Usui, K. Suzuki, T. Kotani, H. Aoki, and K. Kuroki arXiv:1909.00060

\section{Recommended with a Commentary by Atsushi Fujimori, Waseda University}

The recent discovery of superconductivity in the infinite-layer nickel oxide $(\mathrm{Nd}, \mathrm{Sr}) \mathrm{NiO}_{2}$ with the $T_{c}$ of $9-15 \mathrm{~K}$ [1] has invoked tremendous interest since superconductivity has long been sought in two-dimensional (2D) transition-metal oxides other than cuprates and that nickelates have been a most promising candidate. For example, in $\mathrm{LaAlO}_{3} / \mathrm{LaNiO}_{3}$ superlattices, the low-spin $\mathrm{Ni}^{3+}\left(3 d^{7}\right)$ ion has the singly occupied $3 d_{x^{2}-y^{2}}$ orbital strongly hybridizing with $\mathrm{O} 2 p$ orbitals [2] and is, therefore, expected to behave like the $\mathrm{Cu}^{2+}\left(3 d^{9}\right)$ ion in the cuprates having the singly occupied $3 d_{x^{2}-y^{2}}$ orbital strongly hybridizing with $\mathrm{O} 2 p$

The $\mathrm{Ni}^{+}\left(3 d^{9}\right)$ ion in $\mathrm{NdNiO}_{2}$ has the electron configuration identical to that of $\mathrm{Cu}^{2+}$, but hybridization with $\mathrm{O} 2 p$ orbitals is much weaker than in $\mathrm{Cu}^{2+}$ and $\mathrm{Ni}^{3+}$ oxides because of the much larger $\mathrm{O} 2 p$-to-Ni $3 d$ charge-transfer energy of $\Delta \sim 7-9 \mathrm{eV}$ than in the $\mathrm{Cu}^{2+}$ $(\Delta \sim 2-3 \mathrm{eV})$ and $\mathrm{Ni}^{3+}$ oxides $(\Delta<0)[3,4]$. As the on-site Coulomb repulsion at the $\mathrm{Ni}^{+}$site is $U \sim 7 \mathrm{eV}[3],(\mathrm{Nd}, \mathrm{Sr}) \mathrm{NiO}_{2}$ is placed in the Mott-Hubbard regime close to the boundary with the charge-transfer regime of the Zaanen-Sawatzky-Allen diagram [4]. This results in predominant $\mathrm{Ni} d$ character for the doped holes in $(\mathrm{Nd}, \mathrm{Sr}) \mathrm{NiO}_{2}$, in contrast to the predominant oxygen $p$ character for the doped holes in the cuprates. Another consequence of the large $\Delta$ of $\mathrm{NdNiO}_{2}$ is that the $\mathrm{Ni}-\mathrm{Ni}$ superexchange energy $J\left(\propto \Delta^{-2}\right)$ mediated by oxygen should be small: as small as $J \sim 10-30 \mathrm{meV}[4,5]$ compared to the large $J \sim 100-150$ meV for the cuprates. 
The large charge-transfer energy $\Delta$ means the high energy position of the $\mathrm{Ni} 3 d$ levels, which makes their hybridization with the usually empty $\mathrm{Nd} 5 d$ band (or the La $5 d$ band in the case of the closely related $\mathrm{LaNiO}_{2}$ ) important. While the in-plane $\mathrm{Ni} 3 d_{x^{2}-y^{2}}$ orbital does not hybridize with any Nd $5 d$ orbitals [6], the out-of-plane Ni $3 d_{3 z^{2}-r^{2}}$ orbital hybridizes with $\mathrm{Nd}$ $5 d_{3 z^{2}-r^{2}}$ as well as with $\mathrm{Nd} 5 d_{x y}$ (plus interstitial s) orbitals [7]. The Ni $3 d_{3 z^{2}-r^{2}}-\mathrm{Nd} 5 d_{3 z^{2}-r^{2}}$ hybridization creates a small spherical, three-dimensional (3D) electron pocket centered at the $\Gamma$ point of the Brillouin zone (BZ), and the $\mathrm{Ni} 3 d_{3 z^{2}-r^{2}}-\mathrm{Nd} 5 d_{x y}$ hybridization creates another electron pocket at the corners of the 3D BZ [7]. Electrons occupying these pockets originate from the otherwise filled $\mathrm{Ni} 3 d_{3 z^{2}-r^{2}}$ band, resulting in additional holes in the $\mathrm{Ni}$ $3 d$ band: a phenomena called gself-dopingh [8]. The parent compound $\mathrm{NdNiO}_{2}$ [1] as well as $\mathrm{LaNiO}_{2}$ [7] are, therefore, not Mott insulators but metals or poor metals.

A notable physical property of the parent compound $\mathrm{NdNiO}_{2}$ (as well as of $\mathrm{LaNiO}_{2}$ ) is the absence of long-range antiferromagnetic (AFM) order. This has been attributed to AFM spin alignment between the $d_{x^{2}-y^{2}}$ hole and the self-doped $d_{3 z^{2}-r^{2}}$ hole at the same Ni site because of the square-planer effective crystal field [7] or weak electron-electron interaction at the Ni site [9], on the basis of density-functional-theory (DFT) and dynamical mean-fieldtheory studies. Meanwhile, an interesting mechanism for the absence of AFM ordering has been proposed by the first recommended paper and by Refs. [4] and [8]: the local moment of the $\mathrm{Ni}$ ion forms a Kondo singlet with the conduction electrons in the electron pockets rather than an AFM long-range order. In the first recommended paper, Hepting et al. have made extensive soft x-ray spectroscopic studies on $\mathrm{NdNiO}_{2}$, and experimentally confirmed the unusual valence state of $\mathrm{Ni}^{+}$, the weakened $\mathrm{Ni} 3 d-\mathrm{O} 2 p$ hybridization, the Mott-Hubbard character, and the self-doping mechanism mentioned above. However, direct evidence for the Kondo-singlet formation and the Kondo-lattice behavior has been lacking so far.

As for the mechanism of superconductivity, electron-phonon coupling strength estimated from constrained DFT calculation is too weak to explain the observed $T_{c}$ [6]. Most of theoretical studies on the superconductivity in $(\mathrm{Nd}, \mathrm{Sr}) \mathrm{NiO}_{2}$ have been made using $2 \mathrm{D}$ models which take into account electron correlation, namely, the Hubbard and $t$ - $J$ models [10, 11]. In spite of the presence of 3D electron pockets in the parent compound, the electron pockets are expected to shrink with hole doping and the 2D models are be more relevant [12]. In the second recommended paper, Sakakibara et al. report an extensive study of superconductivity in $(\mathrm{Nd}, \mathrm{Sr}) \mathrm{NiO}_{2}$ by treating spin fluctuations within the fluctuation-exchange (FLEX) approximation. A realistic multi-band Hubbard model where all the Ni $3 d$ and Nd $5 d$ bands derived from DFT calculation is employed. A consensus emerges from the theoretical studies that the paring symmetry is $d_{x^{2}-y^{2}}$ and that the $T_{c}$ is lower than those of the cuprates because the energy scale of spin fluctuations is lower than the cuprates due to the smaller superexchange $J$. Sakakibara et al. have explicitly shown that the effect of the electron pockets on superconductivity is minor, reiterating that the 2D models are sufficient to study the superconductivity in the layered nickelates. On the other hand, the sheet resistance at the superconductor-insulator transition (SIT) is significantly larger than $h / 4 e^{2}$, casting doubt on the 2D nature of the superconductivity in $(\mathrm{Nd}, \mathrm{Sr}) \mathrm{NiO}_{2}$ [13].

Four months after the first report of superconductivity in the layered nickelates [1], experimental studies on epitaxial thin films have been limited to the first recommended paper. Even in that work, superconducting samples have not been studies. Experimental studies on bulk $\mathrm{NdNiO}_{2}[5]$ and $(\mathrm{Nd}, \mathrm{Sr}) \mathrm{NiO}_{2}$ samples [14] have been reported, but without 
superconductivity. The authors of Ref. [14] suspect an interfacial origin of superconductivity in the infinite-layer nickelates. There is indeed the possibility that the unusually low valence of $\mathrm{Ni}^{+}$reduces the work function to such an extent that charge transfer from the nickelates to the $\mathrm{SrTiO}_{3}$ substrates induces additional hole doping in the nickelates. The deviation of the sheet resistance from $h / 4 e^{2}$ at the SIT [13] may also be understood if such interfacial superconductivity occurs. Obviously, further material synthesis and characterization studies are necessary to elucidate the mechanism of superconductivity in the nickelates.

\section{References}

[1] D. Li, K. Lee, B. Y. Wang, M. Osada, S. Crossley, H. R. Lee, Y. Cui, Y. Hikita, and H. Y. Hwang, Nature 572, 624 (2019).

[2] J. Chaloupka and G. Khaliullin, Phys. Rev. Lett. 100, 016404 (2008).

[3] A. E. Bocquet, T. Mizokawa, K. Morikawa, and A. Fujimori, S. R. Barman, K. Maiti, D. D. Sarma, Y. Tokura, and M. Onoda, Phys. Rev. B 53, 1161 (1996).

[4] G. A. Sawatzky, Nature 572, 592 (2019); M. Jiang, M. Berciu, and G. A. Sawatzky, arXiv:1909.02557.

[5] Y. Fu, L. Wang, H. Cheng, S. Pei, X. Zhou, J. Chen, S. Wang, R. Zhao, W. Jiang, C. Liu, M. Huang, X. W. Wang, Y. Zhao, D. Yu, S. Wang, and J.-W. Mei: arXiv:1911.03177.

[6] Y. Nomura, M. Hirayama, T. Tadano, Y. Yoshimoto, K. Nakamura, and R. Arita, arXiv:1909.03942.

[7] K.-W. Lee and W. E. Pickett, Phys. Rev. B 70, 165109 (2004).

[8] G.-M. Zhang, Y.-f. Yang, and F.-C. Zhang, arXiv:1909.11845.

[9] Y. Gu, S. Zhu, X. Wang, J. Hu, and H. Chen, arXiv:1911.00814.

[10] X. Wu, D. Di Sante, T. Schwemmer, W. Hanke, H. Y. Hwang, S. Raghu, and R. Thomale, arXiv:1909.03015.

[11] A. S. Botana and M. R. Norman, arXiv:1908.10946.

[12] S. Ryee, H. Yoon, T. J. Kim, M. Y. Jeong, and M. J. Han, arXiv:1909.05824.

[13] S. Uchida, private communication.

[14] Q. Li, C. He, J. Si, X. Zhu, Y. Zhang, and H.-H. Wen, arXiv:1911.02420. 